\title{
Lack of basic and luxury goods and health-related dysfunction in older persons; Findings from the longitudinal SMILE study Daniëlle AI Groffen*1, Hans Bosma' ${ }^{1}$, Marjan van den Akker ${ }^{2}$, Gertrudis IJM Kempen ${ }^{3}$ and Jacques TM van Eijk ${ }^{1}$
}

Address: ${ }^{1}$ School for Public Health and Primary Care (CAPHRI), Department of Social Medicine, Maastricht University, PO Box 616,6200 MD Maastricht, the Netherlands, ${ }^{2}$ School for Public Health and Primary Care (CAPHRI), Department of General Practice, Maastricht University, the Netherlands and ${ }^{3}$ School for Public Health and Primary Care (CAPHRI), Department of Health Care and Nursing Science, Maastricht University, the Netherlands

Email: Daniëlle AI Groffen* - D.Groffen@socmed.unimaas.nl; Hans Bosma - hans.bosma@socmed.unimaas.nl; Marjan van den Akker - Marjan.vandenAkker@hag.unimaas.nl; Gertrudis IJM Kempen - G.Kempen@zw.unimaas.nl; Jacques TM van Eijk - J.vanEijk@socmed.unimaas.nl

* Corresponding author

Published: 17 July 2008

BMC Public Health 2008, 8:242 doi:10.1 I86/147|-2458-8-242
Received: 19 March 2008

Accepted: 17 July 2008

This article is available from: http://www.biomedcentral.com/I47I-2458/8/242

(C) 2008 Groffen et al; licensee BioMed Central Ltd.

This is an Open Access article distributed under the terms of the Creative Commons Attribution License (http://creativecommons.org/licenses/by/2.0), which permits unrestricted use, distribution, and reproduction in any medium, provided the original work is properly cited.

\begin{abstract}
Background: More so than the traditional socioeconomic indicators, such as education and income, wealth reflects the accumulation of resources and makes socioeconomic ranking manifest and explicitly visible to the outside world. While the lack of basic goods, such as a refrigerator, may affect health directly, via biological pathways, the lack of luxury goods, such as an LCD television, may affect health indirectly through psychosocial mechanisms. We set out to examine, firstly, the relevance of both basic and luxury goods in explaining health-related dysfunction in older persons, and, secondly, the extent to which these associations are independent of traditional socioeconomic indicators.
\end{abstract}

Methods: Cross-sectional and longitudinal data from 2067 men and women aged 55 years and older who participated in the Study on Medical Information and Lifestyles Eindhoven (SMILE) were gathered. Logistic regression analyses were used to study the relation between a lack of basic and luxury goods and health-related function, assessed with two sub-domains of the SF- 36 .

Results: The lack of basic goods was closely related to incident physical $(O R=2.32)$ and mental $(O R=2.12)$ dysfunction, even when the traditional measures of socioeconomic status, i.e. education or income, were taken into account. Cross-sectional analyses, in which basic and luxury goods were compared, showed that the lack of basic goods was strongly associated with mental dysfunction. Lack of luxury goods was, however, not related to dysfunction.

Conclusion: Even in a relatively wealthy country like the Netherlands, the lack of certain basic goods is not uncommon. More importantly, lack of basic goods, as an indicator of wealth, was strongly related to health-related dysfunction also when traditional measures of socioeconomic status were taken into account. In contrast, no effects of luxury goods on physical or mental dysfunction were found. Future longitudinal research is necessary to clarify the precise mechanisms underlying these effects. 


\section{Background}

Adverse socioeconomic circumstances affect probabilities of good health and risks of disease [1,2]. Less clear is whether this also holds for older populations of whom many are retired and not in the paid labour force anymore $[3,4]$. In such populations, wealth may be a more valid indicator of socioeconomic ranking than the traditional indicators of socio-economic status, such as education and income. Wealth refers to the individual's or household's total financial resources amassed over the course of life [5]. Hence, the cumulative character of wealth might be important, particularly among older persons because of such life course effects. Furthermore, through being wealthy or not, socioeconomic ranking becomes explicitly visible to the outside world, more than through variations in educational and income level. For example, possession of goods, such as a car and a house, may be an explicit projection of how wealthy a household is.

Although differently measured across studies [5-8], wealth can be measured by the possession or lack of basic (e.g. refrigerator) and luxury goods (e.g. LCD television) [7]. Lack of basic goods may have direct biological effects on health $[7,9]$. For example, the lack of a refrigerator has been shown to increase the risk of stomach cancer [10]. The lack of such basic goods may, further, be related to the lack of sufficient or qualitatively good food and poor housing conditions (e.g. cold and draught), factors that more generally have their sources in the material world [9]. According to some scholars, this neo-material hypothesis is the most important explanation of health differences $[9,11]$.

For the lack of luxury goods in a household, however, it is much more difficult to imagine direct biological effects. The visibility of socioeconomic ranking might be particularly important for the luxury items. The ownership of a large LCD television (preferably visible through the windows) or two cars (preferably both in front of the house) may be an expression of conspicuous consumption and be considered as an outward-directed symbol of status and prestige. The resulting psychosocial comparisons with others (having less or more of such goods) emphasise the potential relevance of psychosocial explanations of socioeconomic differences in health, rather than material explanations alone $[7,12-14]$.

While wealth might be particularly important for an older population because of its cumulative character, the effects of specific measures of wealth on health-related functioning, independent of education and income, have not been widely studied among older persons $[5,15]$. Using crosssectional and longitudinal data from the SMILE study, we set out to examine, firstly, the links between the possession of both basic and luxury goods and both mental and physical dysfunction in older Dutch men and women, and, secondly, the extent to which these links are independent of more traditional socioeconomic indicators, such as education and income.

\section{Methods \\ Design}

Data came from the longitudinal SMILE study (i.e. Study on Medical Information and Lifestyles Eindhoven), which started in November 2002 as a joint project of Maastricht University and the Eindhoven Corporation of Primary Health Care Centres. Eindhoven is a moderate city of approximately 200,000 inhabitants in the Southern part of The Netherlands. General practitioner's registers and annual postal questionnaires were used to collect data on health, lifestyles, and health care use. SMILE is a dynamic cohort, meaning that new participants will enter the population sample when they either reach the age of inclusion or when they are enrolled as a new patient in one of the participating centres and give informed consent. Responders may leave the population either through leaving the participating centres or due to death or drop-out [16]. Persons aged 55 years and older are considered as a separate population within SMILE [17].

\section{Study population}

The present study uses data that were collected in May 2004 and May 2007. In May 2004, 11,180 persons of 55 years or older were sent a self-administered questionnaire, of whom 5,109 (46\%) responded. Forty-two percent $(\mathrm{N}=$ 2,131 ) were followed up until May 2007. Data on educational level was extracted from the May 2003 questionnaire. After exclusion of persons that have missing scores on variables of interest, the main analyses consisted of 2,067 persons (989 men and 1078 women; mean age $=$ 67.6, $\mathrm{SD}=7.5)$.

\section{Ethical review}

Written informed consent was asked when a patient registered in one of the participating health care centers. Privacy regulations are in agreement with the Dutch legislation. The medical ethical committee of the Maastricht Academic Hospital has approved of the study protocol of the SMILE study. Furthermore, the study was registered at the Dutch Data Protection Authority [16].

\section{Measures}

\section{Health-related function}

Information about mental and physical dysfunction was derived from the Dutch version of the MOS SF36 [18,19], assessed in May 2004 and May 2007. The SF36 is a shortform health survey with 36 questions, clustered in eight subscales related to functional health and well-being. The eight scales can be recoded in two distinct higher-order components, i.e. physical and mental function $[19,20]$. 
For the purpose of this study, physical and mental dysfunction was defined as having a score below the 10th percentile (scores of $\leq 30$ and $\leq 36$ out of a range from 10 to 75 , respectively). Furthermore, persons that have more than $50 \%$ missing scores on physical or mental function were excluded from the analyses [19].

\section{Basic and luxury goods}

Basic goods, measured in May 2004, included a freezer, oven, washing machine, refrigerator, telephone, car and own house $[7,17,21]$. Intentionally, three categories were created in such a way that each group contained approximately a third of the sample at baseline: the possession of 0 to 4 basic goods, possession of 5 or 6 basic goods, and possession of all 7 basic goods. Luxury goods have been measured in May 2007 and included a dishwasher, (tumble) dryer, solarium, microwave oven, DVD-player, DVDrecorder, video camera, PC (desktop), laptop, mp3player, internet connection, plasma/LCD television, cellphone, caravan/trailer, second house, musical instrument, second car, navigation system in car, digital television, game console, and a digital photo camera [7]. Again, three categories were created: the possession of 0 to 5 luxury goods; possession of 6 to 10 luxury goods, and possession of 11 to 21 luxury goods.

\section{Covariates}

Covariates were age, gender, educational level, and prevalent severe and less severe disease. Education was measured in May 2003, using a seven-point scale. Three categories were then created in such a way that each group contained approximately a third of the sample: primary school only (lowest); lower vocational education and intermediate general education (middle); intermediate vocational education, higher general education, higher vocational education, and university (highest). Income and financial problems were also separately controlled for, instead of educational level, but as findings were very similar, despite income having more missing scores, only findings for the educational control are presented. Respondents were further asked whether or not they had any of the severe (COPD, heart disease, bowel disease, liver disease, kidney disease, diabetes, cancer, epilepsy, and stroke) and less severe (migraine, joints, rheumatoid arthritis, arthrosis, back, injury, and other) diseases [22]. Presence of diseases was measured in May 2004 and May 2007.

\section{Statistical analyses}

Chi-square tests, based on cross-tabulations, were computed to examine whether there were gender, age, disease status, and physical and mental function differences in the possession of basic or luxury goods. The associations of educational level with the possession of basic and luxury goods and with dysfunction were analysed as well. Multi- ple logistic regression models were fitted to examine whether lack of basic or luxury goods were associated with physical and mental dysfunction (measured in 2007). Possession of all of the selected basic goods or the highest category of luxury goods was the reference category. The first model was adjusted for age and sex. The second model comprised age and gender and was simultaneously adjusted for both the possession of luxury and basic goods. In the third model, odds ratios were further adjusted for educational level. The final model additionally controlled for the presence of severe (0-9) and less severe (0-7) diseases (measured in 2007). For the basic goods, longitudinal analyses were also done to study the relation with incident dysfunction between 2004 and 2007 ( $N=124)$. Prevalent dysfunction at the 2004 baseline was excluded $(\mathrm{N}=150)$. A similar sequence of models as described above was used, adjusting for severe and less severe diseases at baseline (measured in 2004). Finally, exploratory analyses were performed for different subgroups, i.e. younger (55-65) and older (>65) persons, male and female, persons with and without chronic diseases and persons with high or low education. All statistical analyses were performed using SPSS 14.0.1.

\section{Results}

Older persons and women more often reported a relative lack of basic and luxury goods (not tabulated). For example, persons aged 75 or older were more likely to report owning four or less of the basic goods $(20 \%)$ than persons between 55 and 64 years of age (5\%). Furthermore, women were significantly more likely to report owning four or less of the basic goods (11\%) than men (7\%). Similar associations were found for the lack of luxury goods. Seventy percent of persons aged 75 years and older reported owning five or less of the luxury goods, while only $20 \%$ of persons between 55 and 65 years of age did so. Furthermore, only $4 \%$ of the oldest age group reported owning 11 to 21 of the luxury goods, while this was reported by $32 \%$ of the youngest age group. Moreover, men were more likely to report owning 11 to 21 of the luxury goods (22\% versus $15 \%$ ), and were less likely to report having five or less of the luxury goods (30\% versus $45 \%$ ) compared with women.

Table 1 presents the associations between the lack of basic and luxury goods and the prevalence of less severe and more severe diseases and physical and mental dysfunction. Persons owning four or less of the basic goods were more likely to suffer from severe diseases (31\% versus $24 \%$ ) and physical and mental dysfunction (19\% versus $6 \%$ ) than persons owning all of the selected basic goods. The lack of luxury goods was also related to the prevalence of severe diseases and physical and mental dysfunction, although associations were somewhat weaker. 
Table I: Percentages of participants with diseases and with relative poor physical or mental function ${ }^{123}$

\begin{tabular}{|c|c|c|c|c|c|}
\hline & Total & $\geq I$ less severe disease & $\geq I$ severe disease & Physical dysfunction & Mental dysfunction \\
\hline & $N=2858^{4}$ & $N=1139$ & $N=769$ & $N=262$ & $N=239$ \\
\hline \multicolumn{6}{|c|}{ Basic goods } \\
\hline$\leq \mathbf{4}$ & $N=252$ & 42.1 & 31.3 & 19.4 & 18.9 \\
\hline $5-6$ & $N=1566$ & 40.4 & 28.4 & I2.I & 10.6 \\
\hline 7 & $N=1023$ & 39.3 & 24.2 & 6.3 & 5.9 \\
\hline \multicolumn{6}{|c|}{ Luxury goods } \\
\hline$\leq \mathbf{5}$ & $N=1081$ & 39.3 & 31.2 & 16.2 & 12.2 \\
\hline $6-10$ & $N=1232$ & 40.7 & 25.2 & 7.1 & 8.2 \\
\hline||$-2 \mid$ & $N=514$ & $4 I .4$ & 23.5 & 7.2 & 7.0 \\
\hline
\end{tabular}

I Row percentages, for basic and luxury goods separately.

2 Results in bold indicate a significant difference $\left(X^{2} ; p<0.05\right)$.

3 Diseases were self-reported.

${ }^{4}$ Basic and luxury goods have different number of missing values.

Examination of the individual basic items (not tabulated) showed that not owning one's own house (52\%), car $(17 \%)$, or freezer $(16 \%)$ were most often reported. Moreover, the lack of a car and own house were most strongly related to dysfunction. For example, $20 \%$ of persons not owning a car suffered from physical dysfunction compared with $7 \%$ of the car owners. Persons not owning a washing machine were more likely to report mental dysfunction (19\% versus $9 \%$ ) than those who possessed such a machine. Examination of the individual luxury items showed that it was particularly the lack of a personal computer, internet connection, and a mobile phone which were associated with dysfunction. For example, almost $15 \%$ of persons lacking an internet connection at home reported physical dysfunction, compared with only $7 \%$ of persons who had an internet connection available.

There was a significant positive association between the number of basic goods and the number of luxury goods (Spearman's $\mathrm{R}=0.50, \mathrm{P}<0.001$ using the continuous measures). Cross tabulations (not presented) showed that $84 \%$ of persons owning only four or less of the basic goods reported owning only five or less of the luxury goods, compared with only $18 \%$ of persons owning all of the basic goods.
Table 2 shows how education was associated with the possession of basic and luxury goods and health-related dysfunction. Twenty percent of persons with a low educational level reported owning only four or less of the basic goods, while only $4 \%$ of persons with a high educational level did so. Furthermore, $16 \%$ of persons with a low educational level reported poor physical and mental functioning, while only $7 \%$ in the group with a high educational level did so.

Table 3 shows that there was a substantial association between the lack of basic goods and mental dysfunction. Persons with four or less basic goods had more than twice the risk of mental dysfunction $(\mathrm{OR}=2.56)$ of persons owning all of the selected basic goods. The significance of these associations even held after additional adjustment for luxury goods, educational level and severe and less severe diseases $(\mathrm{OR}=2.13)$. The lack of basic goods was also related to physical dysfunction $(\mathrm{OR}=1.91)$. However, the odds ratios became non-significant, when controlled for educational level and diseases. In contrast, the lack of luxury goods was not related to either mental or physical dysfunction. The unadjusted significant association between the relative lack of luxury goods and dysfunction, as shown in Table 1, disappeared when

Table 2: The association of educational level with basic and luxury goods and health-related function ${ }^{123}$

\begin{tabular}{|c|c|c|c|c|c|c|c|c|c|c|}
\hline \multirow{2}{*}{$\begin{array}{l}\text { Educatio } \\
\text { n }\end{array}$} & \multicolumn{3}{|c|}{ Basic goods } & \multicolumn{3}{|c|}{ Luxury goods } & \multicolumn{2}{|c|}{ Physical function } & \multicolumn{2}{|c|}{ Mental function } \\
\hline & $\begin{aligned} & \leq 4 \\
N & =195\end{aligned}$ & $\begin{array}{c}5-6 \\
N=1280\end{array}$ & $7 N=839$ & $\begin{aligned} & \leq 5 \\
N & =868\end{aligned}$ & $\begin{array}{c}6-10 \\
N=1015\end{array}$ & $\begin{array}{c}|I-2| \\
N=4 \mid 7\end{array}$ & $\begin{array}{l}\text { Healthy } \\
N=|87|\end{array}$ & $\begin{array}{c}\text { Poor } \\
\mathbf{N}=208\end{array}$ & $\begin{array}{l}\text { Healthy } \\
N=1893\end{array}$ & $\begin{array}{c}\text { Poor } \\
N=185\end{array}$ \\
\hline Low & 19.8 & 67.4 & 12.8 & 65.2 & 28.7 & 6.0 & 84.2 & 15.8 & 84.2 & 15.8 \\
\hline Middle & 8.5 & 60.8 & 30.8 & 41.0 & 42.9 & 16.1 & 89.1 & 10.9 & 91.4 & 8.6 \\
\hline High & 3.9 & 45.7 & 50.5 & 24.0 & 51.3 & 24.7 & 92.7 & 7.3 & 93.1 & 6.9 \\
\hline
\end{tabular}

\footnotetext{
I Row percentages.

2 Results in bold indicate a significant difference $\left(X^{2} ; p<0.05\right)$.

3 Basic and luxury goods have different number of missing values.
} 
Table 3: Adjusted Odds Ratios (OR) of physical and mental dysfunction by basic and luxury goods

\begin{tabular}{|c|c|c|c|c|}
\hline & Model I I OR (95\% Cl) & Model $2^{2}$ OR $(95 \% \mathrm{Cl})$ & Model $3^{3}$ OR $(95 \% \mathrm{Cl})$ & Model 44 OR $(95 \% \mathrm{Cl})$ \\
\hline Basic goods & \multicolumn{4}{|c|}{ Physical dysfunction $(N=2067)$} \\
\hline$\leq 4$ & $1.91(1.10-3.33)$ & $1.65(0.93-2.93)$ & $1.49(0.83-2.68)$ & $1.28(0.68-2.42)$ \\
\hline $5-6$ & $1.62(1.15-2.29)$ & $1.51(1.06-2.15)$ & $1.42(0.99-2.04)$ & I.4I (0.96-2.08) \\
\hline 7 & 1.00 & 1.00 & 1.00 & 1.00 \\
\hline \multicolumn{5}{|c|}{ Luxury goods } \\
\hline$\leq 5$ & $1.54(0.94-2.52)$ & $1.32(0.79-2.19)$ & $1.25(0.75-2.09)$ & I.II (0.64-I.92) \\
\hline $6-10$ & $0.88(0.55-1.42)$ & $0.82(0.51-1.32)$ & $0.81(0.50-1.31)$ & $0.77(0.46-1.28)$ \\
\hline$|1-2|$ & 1.00 & 1.00 & 1.00 & 1.00 \\
\hline Basic goods & \multicolumn{4}{|c|}{ Mental dysfunction $(N=2067)$} \\
\hline$\leq 4$ & $2.56(1.48-4.34)$ & $2.47(I .39-4.4 I)$ & $2.18(1.21-3.93)$ & $2.13(1.17-3.84)$ \\
\hline $5-6$ & $1.53(1.08-2.18)$ & I.5। (1.05-2.17) & $1.39(0.96-2.02)$ & $1.37(0.95-1.99)$ \\
\hline 7 & 1.00 & 1.00 & 1.00 & 1.00 \\
\hline \multicolumn{5}{|l|}{ Luxury goods } \\
\hline$\leq 5$ & $1.33(0.82-2.16)$ & $1.04(0.62-1.74)$ & $0.97(0.58-1.62)$ & $0.91(0.54-1.53)$ \\
\hline $6-10$ & $1.02(0.65-1.60)$ & $0.91(0.58-1.44)$ & $0.90(0.57-1.43)$ & $0.88(0.56-1.40)$ \\
\hline||$-2 \mid$ & 1.00 & 1.00 & 1.00 & 1.00 \\
\hline
\end{tabular}

I Model $\mathrm{I}$ is adjusted for age and gender.

2 Model 2 is adjusted for age, gender and simultaneously for luxury goods or basic goods.

3 Model 3 is additionally adjusted for education.

${ }_{4}^{4}$ Model 4 is additionally adjusted for severe and less severe diseases.

controlled for age and sex. Those without luxury goods being older, in particular, explained $85 \%$ of the corresponding unadjusted odds ratio of 2.59 (95\% CI: 1.664.04).

Longitudinal analyses showed that the lack of basic goods was related to both incident mental and physical dysfunction, even after additional adjustment for gender, age, luxury goods, educational level, and severe and less severe disease $(\mathrm{OR}=2.32$ for physical functioning and $\mathrm{OR}=2.12$ for mental functioning) (Table 4).

Effects were similar in men and women, for the younger $(\leq 65)$ and older $(>65)$ age groups, for diseased and nondiseased persons, and for both the lower and higher educated persons, as the respective interaction terms were not significant in our analyses. Furthermore, when using linear regression analyses with mental and physical function as continuous variables, similar associations were found.

\section{Discussion}

In this group of older Dutch men and women, a lack of basic goods was not uncommon. Moreover, this lack of basic goods, as indicator of wealth, turned out to be a good predictor of both incident physical and mental dysfunction, even when traditional measures of socio-economic status, i.e. education and income, were taken into account. No significant associations were found between the lack of luxury goods and dysfunction. All association were independent of the prevalence of severe and less severe diseases.

Table 4: Odds ratios (OR) of the incidence of physical and mental dysfunction by basic goods 1

\begin{tabular}{|c|c|c|c|c|}
\hline & Model I2 OR ( $95 \% \mathrm{Cl})$ & Model $2^{3}$ OR $(95 \% \mathrm{Cl})$ & Model $3^{4}$ OR $(95 \% \mathrm{Cl})$ & Model $4^{5}$ OR $(95 \% \mathrm{Cl})$ \\
\hline Basic goods & \multicolumn{4}{|c|}{ Physical functioning $(n=1769)$} \\
\hline$\leq 4$ & $2.56(1.17-5.60)$ & $2.52(1.11-5.70)$ & $2.63(1.14-6.07)$ & $2.32(1.00-5.38)$ \\
\hline $5-6$ & $1.73(1.04-2.89)$ & $1.71(1.01-2.90)$ & $1.76(1.03-3.00)$ & $1.70(0.99-2.90)$ \\
\hline 7 & 1.00 & 1.00 & 1.00 & 1.00 \\
\hline Basic goods & \multicolumn{4}{|c|}{ Mental functioning $(n=\mid 761)$} \\
\hline$\leq 4$ & $2.50(1.26-4.94)$ & $2.52(1.23-5.16)$ & $2.30(1.11-4.79)$ & $2.12(1.01-4.73)$ \\
\hline $5-6$ & $1.35(0.87-2.11)$ & $1.36(0.86-2.15)$ & $1.29(0.8 \mathrm{I}-2.05)$ & $1.26(0.79-2.01)$ \\
\hline 7 & 1.00 & 1.00 & 1.00 & 1.00 \\
\hline
\end{tabular}

\footnotetext{
I Prevalent dysfunction cases in 2004 are excluded from the analyses.

2 Model I is adjusted for age and gender.

3 Model 2 is additionally adjusted for luxury goods.

${ }^{4}$ Model 3 is additionally adjusted for education.

${ }^{5}$ Model 4 is additionally adjusted for severe and less severe diseases.
} 
Given that basic goods are indicators of wealth [5-8], our results suggest that for an older population wealth might be an additional or even more appropriate predictor of health-related dysfunction than the traditional measures of SES, i.e. education and income. This may be due to its cumulative character, indicating economic advantage and disadvantage amassed over the course of life. Wealth may, further, buffer the effects of lost or temporarily low income $[5,15]$.

The lack of basic goods having more impact on healthrelated dysfunction than the lack of luxury goods is consistent with the view that material factors are important determinants of health. Living in poor material conditions or with a lack of resources may have a direct, biological, effect on health [9]. Furthermore, the lack of a car makes shopping for (healthy) food and access to health care services much more difficult and the ownership of a house may, on average, be associated with a better housing conditions [23].

However, psychological or psychosocial pathways cannot be excluded either. The lack of a car, refrigerator, or oven is visible for neighbours, friends, and acquaintances. The negative social comparison resulting from this apparent visibility might have adverse effects on self-esteem and subjective prestige, pride, and status through which eventually both mental and physical dysfunction might become compromised as well $[12,14,24]$. The possibility that psychological pathways might contribute is also substantiated by recent experiences with Dutch food banks. These are increasingly being moved to the suburbs, where their customers report less shame and other psychosocial problems, given that their visits to these more secluded environments are less likely to be noticed [25]. Perhaps shame may even be more prominent when lacking sufficient food or a refrigerator than a second house or a DVD recorder.

Why then are there no effects of a lack of luxury goods, where visibility and corresponding psychosocial pathways were hypothesised to have particular relevance? Our data suggest that the possession of a large LCD television or a second car does not make older persons healthier and happier compared with their neighbours who do not have such items. Pikhart and colleagues (2003), however, found that luxury items remained strongly associated with self-rated health even in fully adjusted models. It should, however, be noticed that this finding only held for a, on average younger (18+) Hungarian population. Similar to our study, luxury goods were only studied using cross-sectional data. Future longitudinal research is necessary to clarify the relations. As mentioned above, visible status, social comparisons and related psychosocial mechanisms [26] might also, and perhaps particularly, hold for explaining the adverse health outcomes of lacking basic goods.

\section{Methodological considerations}

Several methodological limitations may affect the interpretation of the results of our study. Firstly, the selection of items is cultural [27] and time-dependent. The individual basic and luxury good items were chosen on the basis of recent reports $[7,21]$. Furthermore, as we state in our results section, certain items have more predictive power than others. However, before reducing the scales to the items that are predictive for dysfunction, more research is needed, also with other health outcomes. Further development and validation of measurement instruments to assess the possession of both basic and luxury goods is recommended. Moreover, more attention should be paid to additional measures of poor material circumstances, such as poor physical housing and working conditions (e.g. dampness, mould, and cold in the house and lifting heavy loads at work) $[28,29]$.

Secondly, our study relied solely on self-reports which might have introduced measurement error for the dysfunction measures [30]. Individuals with a general tendency towards negative perceptions of material well-being may also over-report symptoms of health-related dysfunction (i.e. negative affectivity) [31]. This may have led to an overestimation of the presented association. However, by excluding prevalent dysfunction cases from the longitudinal analyses, persons with negative affectivity $[32,33]$ have also been excluded. Overlap between the physical and mental functioning component of the SF-36 outcome measure might also have distorted our results [34]. However, these measures were only weakly correlated (Pearson's $\mathrm{R}=0.168, \mathrm{p}<0.001$ ) within our study population. Furthermore, poor people may have underreported their poverty. Out of shame, people may not want to admit not being able to afford certain (basic) items. This bias may have led to an underestimation of the presented associations.

Thirdly, the question remains why the longitudinal association between basic goods and physical dysfunction is stronger than the corresponding cross-sectional association. Except that longitudinal analyses are generally considered superior because of the exclusion of reverse causation, we could not come to an answer to this question. More research is recommended to disentangle possible underlying mechanisms.

Finally, our research may be limited by potential selection biases. Older persons living in nursing homes were not included which restricts the generalisability of our findings. The most disadvantaged older persons may be underrepresented in our research, because of premature 
mortality (the 'wealthy survivor' effect). Response analyses showed that men and persons from the youngest (<65) age groups were more likely to respond. Furthermore, attrition (between 2004 and 2007, 33\% were lost to follow-up) was higher for persons that were lower educated and had worse scores on physical and mental function scores at the 2004 baseline $(\mathrm{P}<0.001)$. Missing values analyses revealed that persons with missing values on mental and/or physical function scales were significantly more likely to be lower educated, have fewer basic and luxury goods and have worse physical and mental function scores at the 2004 baseline. Similarly, persons with missing values on basic or luxury good items were more likely to report poor mental and physical function. This pattern of selective response and attrition may have led to an underestimation of the reported associations, although attrition's effect may be more disturbing for descriptive results than for measures of (longitudinal) association [35].

\section{Conclusion}

Even in a relatively wealthy country like the Netherlands, the lack of certain basic goods is not uncommon. More importantly, lack of basic goods, as indicator of wealth, was strongly related to health-related dysfunction, also when taking into account traditional measures of socioeconomic status. In contrast, no effects of luxury goods on physical or mental dysfunction were found. Future longitudinal research is necessary to clarify the precise mechanisms underlying the effects and -particularly in older persons- to explore strategies to intervene upon the adverse effects of material deprivation.

\section{Competing interests}

The authors declare that they have no competing interests.

\section{Authors' contributions}

DAIG performed the statistical analyses and drafted the manuscript. HB formulated the hypotheses. MvdA was responsible for the design of the SMILE study. All authors (HB,MvdA,GIJMK,JTMvE) edited and approved the final manuscript.

\section{Acknowledgements}

The longitudinal SMILE study is carried out by the department of General Practice of Maastricht University, in collaboration with the Public Health Services of the city of Eindhoven. Currently, nine general practices and their 40,000 patients are participating. The investigators are indebted to the participants for their willingness to participate in the study.

\section{References}

I. Wilkinson RG, Marmot M: Social determinants of health: the solid facts. 2nd edition. WHO; 2003.

2. Ferrie JE, Shipley MJ, Davey Smith G, Stansfeld S, Marmot M: Change in health inequalities among British civil servants: the Whitehall II study. Journal of Epidemiology and Community Health 2002, 56:922-926.
3. Breeze E, Fletcher AE, Leon DA, Marmot MG, Clarke RJ, Shipley MJ: Do socioeconomic disadvantages persist into old age? Selfreported morbidity in a 29-year follow-up of the Whitehall study. American Journal of Public Health 200I, 9 I (2):277-283.

4. Artazcoz L, Rueda S: Social inequalities in health among the elderly: a challenge for public health research. Journal of Epidemiology and Community Health 2007, 61:466-467.

5. Pollack CE, Chideya S, Cubbin C, Williams B, Dekker M, Braveman PA: Should health studies measure wealth? A systematic review. American Journal of Preventive Medicine 2007, 33(3):250-264.

6. Macintyre S, McKay L, Der G, Hiscock R: Socio-economic position and health: what you observe depends on how you measure it. Journal of Public Health Medicine 2003, 25(4):288-294.

7. Pikhart $\mathrm{H}$, Bobak M, Rose R, Marmot M: Household item ownership and self-rated health: material and psychosocial explanations. BMC Public Health 2003, 3(38):.

8. Duncan G], Daly MC, McDonough P, Williams DR: Optimal indicators of socioeconomic status for health research. American Journal of Public Health 2002, 92(7): I I5 I-I I 57.

9. Lynch JW, Davey Smith G, Kaplan GA, House JS: Income inequality and mortality: importance to health of individual income, psychosocial environment, or material conditions. British Medical Journal 2000, 320: I200-1 204.

10. Boeing $\mathrm{H}$ : Epidemiological research in stomach cancer: progress over the last ten years. Journal of Cancer Research and Clinical Oncology 199I, I I7(2): 133-143.

II. Macleod J, Davey Smith G, Metcalfe C, Hart C: Is subjective social status a more important determinant of health than objective social status? Evidence from a prospective observational study of Scottish men. Social Science and Medicine 2005, 6I(9): 1916-1929.

12. Marmot MG, Wilkinson RG: Psychosocial and material pathways in the relation between income and health: a response to Lynch et al. British Medical Journal 200I, 322:1233-1236.

13. Marmot MG: Understanding social inequalities in health. Perspect Biol Med 2003, 46(3 Suppl):S9-23.

14. Fliessbach K, Weber B, Trautner P, Dohmen T, Sunde U, Elger CE, Falk $A$ : Social comparison affects reward-related brain activity in the human ventral striatum. Science 2007, 3 I 8: | 305- I 308.

15. von dem Knesebeck O, Luschen G, Cockerham WC, Siegrist J: Socioeconomic status and health among the aged in the United States and Germany: A comparative cross-sectional study. Social Science and Medicine 2003, 57:1643-1652.

16. Van den Akker M, Spigt MG, de Raeve L, van Steenkiste B, Metsemakers JFM, van Voorst Ej, de Vries H: The SMILE study: A Study of Medical Information and Lifestyles in Eindhoven. The rationale and contents of a large prospective dynamic cohort study. BMC Public Health 2008, 8(19):.

17. Groffen DAI, Bosma $\mathrm{H}$, van den Akker M, Kempen GIJM, van Eijk JTM: Material deprivation and health-related dysfunction in older Dutch people; Findings from the SMILE study. European Journal of Public Health 2008, I 8(3):258-263.

18. Ware JE, Sherbourne CD: The Rand-36 Short-form Health Status Survey I: Conceptual framework and item selection. Medical Care 1992, 30(6):473-48I.

19. Van der Zee KI, Sanderman R, Heyink J: De psychometrische kwaliteiten van de MOS 36 item Short Form Health Survey in een Nederlandse populatie. Tijdschrift voor Sociale Gezondheidszorg 1993, 4:|83-19|.

20. Ware JE, Kosinski M: Interpreting SF-36 summary health measures: a response. Quality of Life Research 200I, 10:4I5-420.

21. Jehoel-Gijsbers G: Sociale uitsluiting in Nederland. The Hague, Netherlands Institute for Social Research/SCP; 2003.

22. Koster A, Bosma H, Kempen GI, Van Lenthe FJ, Van Eijk JT, Mackenbach JP: Socioeconomic inequalities in mobility decline in chronic disease groups (asthma/COPD, heart disease, diabetes mellitus, low back pain): only a minor role for disease severity and comorbidity. Journal of Epidemiology and Community Health 2004, 58:862-869.

23. Grundy H, Holt G: The socioeconomic status of older adults: how should we measure it in studies of health inequalities? Journal of Epidemiology and Community health 200I, 55:895-904.

24. Vetter S, Endrass J, Schweizer I, Teng HM, Rossler W, Gallo WT: The effects of economic deprivation on psychological well-being among the working population of Switzerland. BMC Public Health 2006, 6(I):223. 
25. Desain L, Van Gent MJ, Kroon P, Langendijk F, Van Waveren B: Eindrapport klantenanalyse voedselbanken. Amsterdam, the Netherlands, Regioplan beleidsonderzoek; Ministry of Social Affairs; 2006.

26. Wilkinson RG, Pickett KE: The problems of relative deprivation: Why some societies do better than others. Social Science and Medicine 2007, 65(9): 1965-1978.

27. Dressler WW: Culture and the risk of disease. British Medical Bulletin 2004, 69:2I-3I.

28. Evans J, Hyndman S, Stewart Brown S, Smith D, Petersen S: An epidemiological study of the relative importance of damp housing in relation to adult health. Journal of Epidemiology and Community Health 2000, 54(9):677-686.

29. Packer CN, Stewart Brown S, Fowle SE: Damp housing and adult health - results from a life-style study in Worcester, England. Journal of Epidemiology and Community Health 1994, 48:555-559.

30. Kempen GI, Steverink N, Ormel J, Deeg DJ: The assessment of ADL among frail elderly in an interview survey:self-report versus performance-based tests and determinants of discrepancies. J Gerontol B Psychol Sci Soc Sci 1996, 5 I(5):P254-260.

31. Macleod J, Davey Smith G, Heslop P, Metcalfe C, Carroll D, Hart C: Psychological stress and cardiovascular disease: empirical demonstration of bias in a prospective observational study of Scottish men. British Medical Journal 2002, 324: I247-I253.

32. Kressin NR, Spiro A, Skinner KM: Negative affectivity and health related quality of life. Medical Care 2000, 38(8):858-867.

33. Watson D, Pennebakker JW: Health complaints, stress, and distress: exploring the central role of negative affectivity. Psychological Review 1989, 96(2):234-254.

34. Simon GE, Revicki DA, Grothaus L, Vonkorff M: SF-36 summary scores; Are physical and mental health truly distinct? Medical Care 1998, 36(4):567-572.

35. Kempen GIJM, Van Sonderen E: Psychological attributes and changes in disability among low-functioning, older persons: does attrition affect the outcomes? Journal of Clinical Epidemiology 2002, 55:224-229.

\section{Pre-publication history}

The pre-publication history for this paper can be accessed here:

http://www.biomedcentral.com/1471-2458/8/242/pre

pub

Publish with Bio Med Central and every scientist can read your work free of charge

"BioMed Central will be the most significant development for disseminating the results of biomedical research in our lifetime. "

Sir Paul Nurse, Cancer Research UK

Your research papers will be:

- available free of charge to the entire biomedical community

- peer reviewed and published immediately upon acceptance

- cited in PubMed and archived on PubMed Central

- yours - you keep the copyright

Submit your manuscript here:

http://www.biomedcentral.com/info/publishing_adv.asp
BioMedcentral 